\title{
Analysis of the Cherenkov Telescope Array first Large-Sized Telescope real data using convolutional neural networks
}

Thomas Vuillaume $^{a}$, Mikaël Jacquemont ${ }^{a, b}$, Mathieu de Bony de Lavergne ${ }^{a, *}$, David A. Sanchez ${ }^{a}$, Vincent Poireau ${ }^{a}$, Gilles Maurin $^{a}$, Alexandre Benoit $^{b}$, Patrick Lambert $^{b}$, Giovanni Lamanna ${ }^{a}$ on behalf of the CTA LST project Collaboration

(a complete list of authors can be found at the end of the proceedings)

${ }^{a}$ Laboratoire d'Annecy de Physique des Particules, Univ. Grenoble Alpes, Univ. Savoie Mont Blanc, CNRS, LAPP, 9 chemin de bellevue, 74940 Annecy, France

${ }^{b}$ LISTIC, Université Savoie Mont-Blanc Polytech

Annecy-Chambéry, 5 chemin de bellevue, 74940 Annecy, France

E-mail: thomas.vuillaume@lapp.in2p3.fr

The Cherenkov Telescope Array (CTA) is the future ground-based gamma-ray observatory and will be composed of two arrays of imaging atmospheric Cherenkov telescopes (IACTs) located in the Northern and Southern hemispheres respectively. The first CTA prototype telescope built on-site, the Large-Sized Telescope (LST-1), is under commissioning in La Palma and has already taken data on numerous known sources. IACTs detect the faint flash of Cherenkov light indirectly produced after a very energetic gamma-ray photon has interacted with the atmosphere and generated an atmospheric shower. Reconstruction of the characteristics of the primary photons is usually done using a parameterization up to the third order of the light distribution of the images. In order to go beyond this classical method, new approaches are being developed using state-of-the-art methods based on convolutional neural networks (CNN) to reconstruct the properties of each event (incoming direction, energy and particle type) directly from the telescope images. While promising, these methods are notoriously difficult to apply to real data due to differences (such as different levels of night sky background) between Monte Carlo (MC) data used to train the network and real data. The GammaLearn project, based on these CNN approaches, has already shown an increase in sensitivity on MC simulations for LST-1 as well as a lower energy threshold. This work applies the GammaLearn network to real data acquired by LST-1 and compares the results to the classical approach that uses random forests trained on extracted image parameters. The improvements on the background rejection, event direction, and energy reconstruction are discussed in this contribution.

$37^{\text {th }}$ International Cosmic Ray Conference (ICRC 2021)

July 12th - 23rd, 2021

Online - Berlin, Germany

*Presenter 


\section{Introduction}

The Cherenkov Telescope Array (CTA) is the future of ground-based gamma-ray astronomy. With two arrays built on two sites, La Palma (Canary Islands) and Paranal (Chile), it will be composed of tens of imaging atmospheric Cherenkov telescopes (IACT) of different sizes that will increase the sensitivity by a factor of 5 to 10 compared to the current generation of instruments in the range from $20 \mathrm{GeV}$ to $300 \mathrm{TeV}$. The Large-Sized Telescope 1 (LST-1) is the first CTA prototype telescope built on-site (at the Observatorio del Roque de los Muchachos, La Palma) and is currently under commissioning. Thanks to its size ( $23 \mathrm{~m}$ mirror diameter), it is specially designed to focus on the low energy range of CTA (from $20 \mathrm{GeV}$ to a few hundreds of $\mathrm{GeV}$ ). Data from several known gamma-ray sources have already been acquired.

Relativistic particles (either gamma-rays or cosmic rays) entering the atmosphere produce atmospheric showers, whose sub-products produce Cherenkov light collected by IACTs. A complex analysis, also called event reconstruction, is necessary to determine the particle type (separating gamma rays from cosmic rays which represent the background), its energy and incoming direction from the images produced by IACTs. We present this analysis in further details in section II and then explain how it can benefit from convolutional neural networks (CNNs), comparing the standard approach and our network $\gamma$-PhysNet DA. We present performances on simulations in section III and on LST-1 data in section IV.

\section{Event reconstruction}

The Cherenkov light generated by atmospheric showers is collected on the ground by the LST1 optical system that focuses it to an ultra-fast and sensitive camera sampling the signal into a temporal series of 40 snapshots of 1855 pixels of $1 \mathrm{~ns}$ each (also called waveform). This process is depicted in Figure 1. The waveform is then calibrated and integrated to obtain two images, one containing the pixel charges and one the mean arrival time of the Cherenkov photon in each pixel. These two images are then analysed to extract image parameters used to reconstruct the particles physical parameters of interest (type, energy, incoming direction) using machine learning algorithms such as random forests [1].

Thanks to accurate Monte Carlo simulations $[2,3]$, machine learning algorithms are trained on these images in order to reconstruct the corresponding physical parameters. The algorithms can then be applied to simulated test datasets in order to compute the instrument response function, and

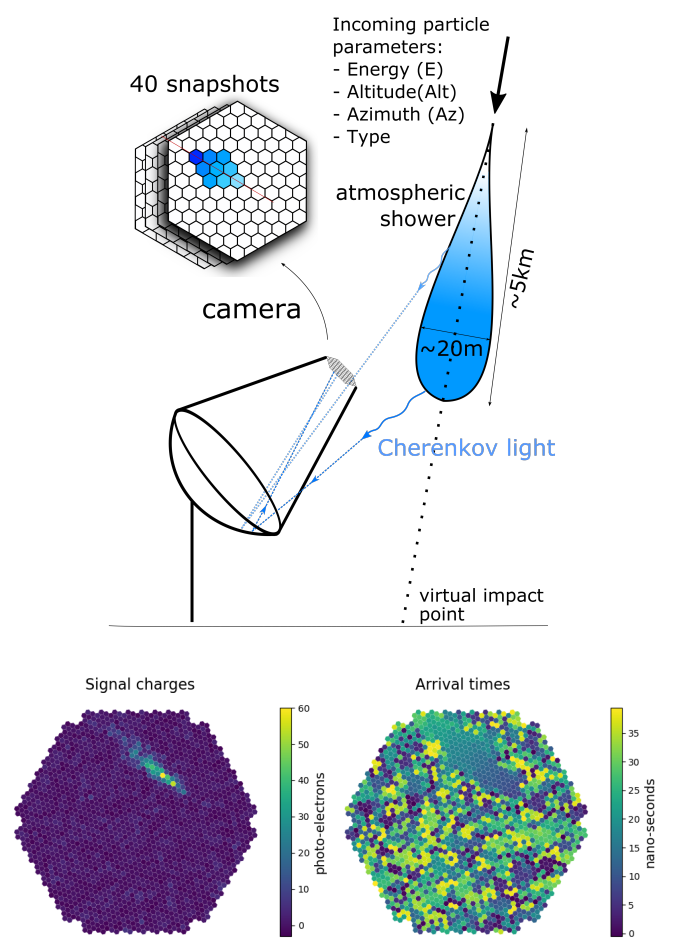

Fig. 1: Top: LST-1 observation principle. Bottom: Extracted signal charges and times. so the performance of the reconstruction, or to data from observations. 
The steps of image parameters extraction, training, and inference on observations is the one we propose to enhance with deep learning. Indeed, since the AlexNet revolution [4] CNNs have shown in many fields [5] their capacity to replace and overtake solutions with engineered parameters extraction. They have been applied to IACT event reconstruction in the past on CTA simulated data [6-8] and on H.E.S.S. [9, 10] data, but with limited success on observations. Here we will compare the standard approach using engineered parameters and random forests with the CNN we developed specifically for the tasks at hand, $\gamma$-PhysNet DA. The first step of calibration plus charge integration is common for both approaches and presented in further details in [11]. It is performed using the lstchain v0.7.3 library [12] based on ctapipe v10.5 $[13,14]$.

\section{II-A LST-1 classical approach: Hillas and ensemble models}

A classical approach, called Hillas by the name of its creator [15], is based on characteristic parameters extracted from these images. This is the standard analysis method in use for LST1 data analysis. The images first go through a cleaning step called tailcut-cleaning requiring pixels to be above a given picture threshold and a neighbor above a second threshold. The same mask is then applied on the temporal map. Image parameters are then extracted from these cleaned images. The parameters (defined in lstchain v0.7.3) used in this work are: image intensity, ellipsoid width, length, position and orientation in the camera, second order moments, signal time development gradient and charges containment.

Three different random forests (RF) are then trained, one for each task: particle classification, energy reconstruction and direction reconstruction. The performances of these RFs are presented in section III. This approach, later noted Hillas $+R F$, will serve as the reference for the rest of this work. The complete set of parameters is given in lstchain v0.7.3 standard configuration.

\section{II-B $\gamma$-PhysNet - a convolutional neural network to analyse LST-1 data}

The CNN we developed, $\gamma$-PhysNet DA, is presented in depth in Ref. [16, 17]. It is a multi-task network, so contrary to the classical approach and to previous works using CNNs, a single algorithm is trained to perform the full event reconstruction (particle classification, energy reconstruction and direction reconstruction). It is composed of a first convolution block common to all tasks and based on ResNet-56 [18] enhanced with indexed convolutions [19] and Dual Attention mechanism [20].

\section{Performance comparison on simulated data}

The Monte Carlo simulations, produced using CORSIKA [2] and simtel_array [3] are finetuned to the LST-1 at a pointing of $20^{\circ}$ zenith in the South direction. The training dataset is composed of isotropic gamma rays and protons while the test dataset is composed of point-source gammas at an offset of $0.4^{\circ}$ to the camera center, and isotropic protons and electrons.

Quality cuts are applied to select events: the integrated charge after image cleaning must be greater than 50 photoelectrons, the fraction of that integrated charge in the outermost ring of pixels in the camera must be below $20 \%$ and the image must pass a tailcut cleaning with a picture threshold of 6 photoelectrons and a neighbor threshold of 3 photoelectrons. Note however that 
the cleaning itself is applied only in the case of the Hillas+RF procedure, while the entire image information is kept in the case of $\gamma$-PhysNet DA.

\section{III-A Instrument response functions}

Applying the trained algorithms to the test dataset, one can compute the instrument response functions (Figure 2, as defined in Ref. [21]) for the two approaches. The gammaness and angular cuts are determined for best sensitivity in each energy bin using the pyirf v0.4 package [22]. The highest performances of $\gamma$-PhysNet DA on all tasks are clearly demonstrated at all energies and in particular at the lowest ones (below $300 \mathrm{GeV}$ ). The greater classification power of $\gamma$-PhysNet $\mathrm{DA}$ is shown by the higher effective area and by the higher area under the roc curve.
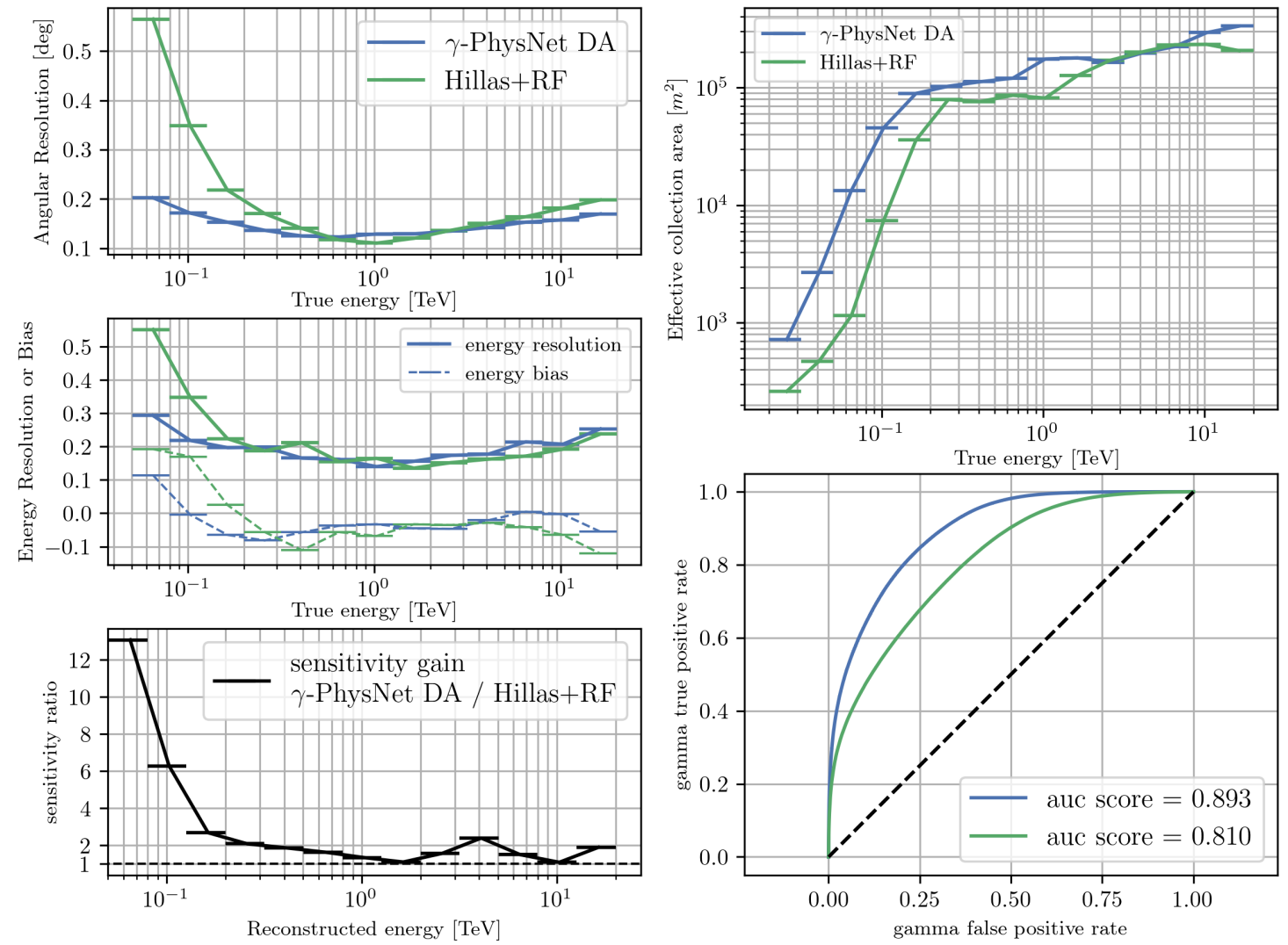

Fig. 2: Comparison of instrument response functions and performances on simulated data between the reference (Hillas+RF) and our network $\gamma$-PhysNet DA. Top-left panels: angular resolution, energy resolution and energy bias (lower is better). Bottom-left: Sensitivity ratio (higher is better). Top-right: effective area (higher is better). Bottom-right: ROC curve (higher auc score is better).

\section{Application to LST-1 data}

\section{IV-A Methodology}

The fairest way to compare the performances of two reconstruction chains is to match their background levels. To do so, we first apply the quality selection cuts common to both chains. 


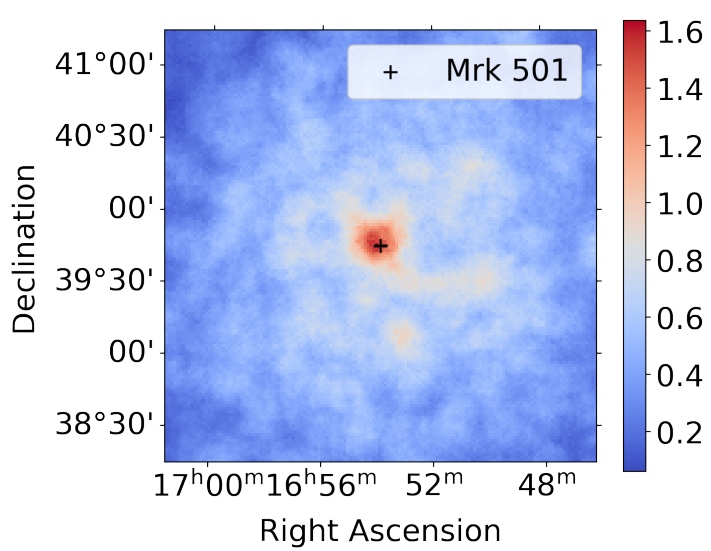

(a) Hillas $+\mathrm{RF}$

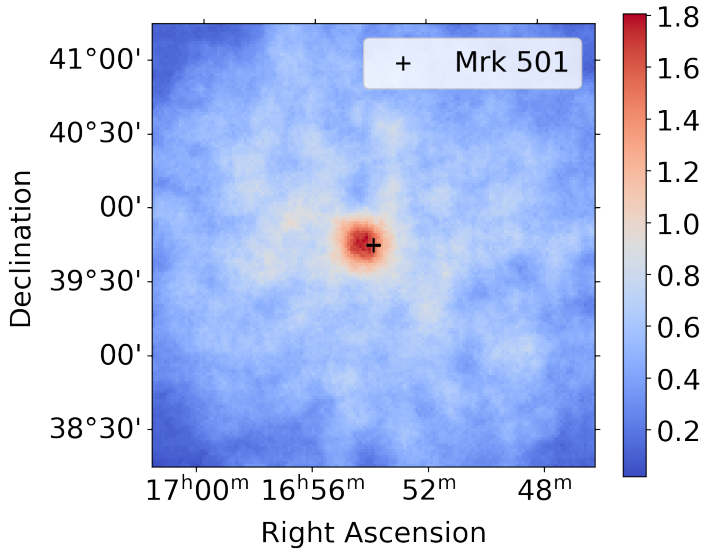

(b) $\gamma$-PhysNet DA

Fig. 3: Smoothed (kernel=0.12) count map of gamma-like events around Markarian 501.

Then, we fix the gammaness (likelihood of an event to be a gamma) cut of the standard Hillas+RF analysis at a given level and the gammaness cut of $\gamma$-PhysNet DA is then determined in order to get the same background level as the one of the reference in each energy bin in the background region (determined with the multiple OFF technique [23]). It is worth noticing that several values have been tested for the reference gammaness cut with no significant change in the results - only one analysis has been kept for simplicity.

\section{IV-B Detection of Markarian 501}

Markarian 501 is a well-known blazar at $\mathrm{TeV}$ energy range. The observation dataset is composed of 4 runs taken on the night of the 20 March 2021 for a total of $1 \mathrm{~h}$. Observed zenith angles range from $20^{\circ}$ to $12.5^{\circ}$. All observations are conducted in wobble mode with an offset of $0.4^{\circ}$.

In this case, the analysis (training and inference) has been performed with cleaning levels of $(8,4)$. The high-level analysis is performed using gammapy v0.18.2 [24], the standard science tool for CTA. Background estimation is done with the reflected multiple off technique [23]. The excess and the background are calculated per reconstructed energy bin.

First a counts map of gamma-like events is generated for both chains (Fig. 3). For $\gamma$-PhysNet DA and Hillas+RF we can notice a hot spot at the source position, later confirmed by proper background estimation and significance calculation. Figure 4 shows the events counts as a function of the separation angle to the source for both chains in OFF and ON regions. On these figures one can observe a bias in the direction reconstruction in the case of $\gamma$-PhysNet DA. The reason of this bias on observations needs further investigation but preliminary studies show an impact of the discrepancy in pointing direction between simulations and observations. An angular cut of $\theta=0.2 \mathrm{deg}$ is applied on data to select the source region in agreement with the average angular resolution and standard cuts applied in LST-1 data analysis [21].

Figure 5 presents a comparative excess count as a function of the reconstructed energy. We can notice the greater excess of gamma-like events detected by $\gamma$-PhysNet DA compared to the standard Hillas+RF approach, especially at low energies (around $100 \mathrm{GeV}$ ). This is in harmony 
with the results obtained on Monte Carlo simulations presented in figure 2, even though the performance gain is not as large.

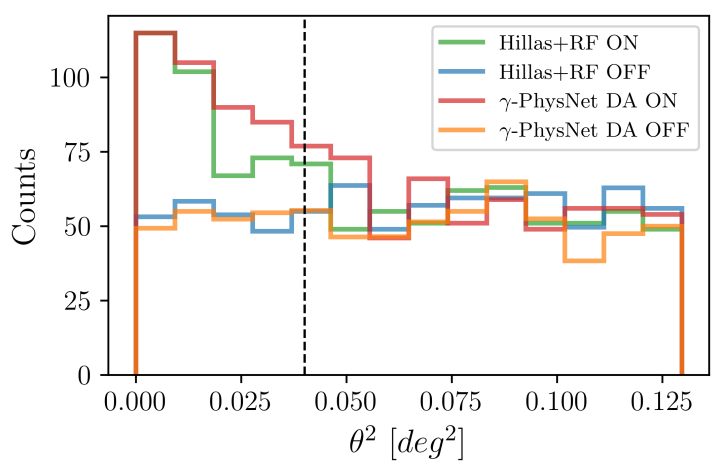

Fig. 4: Event count as a function of the squared angular separation to Mrk 501. The vertical dashed line represent the $\theta^{2}$ cut applied on data for the analysis.

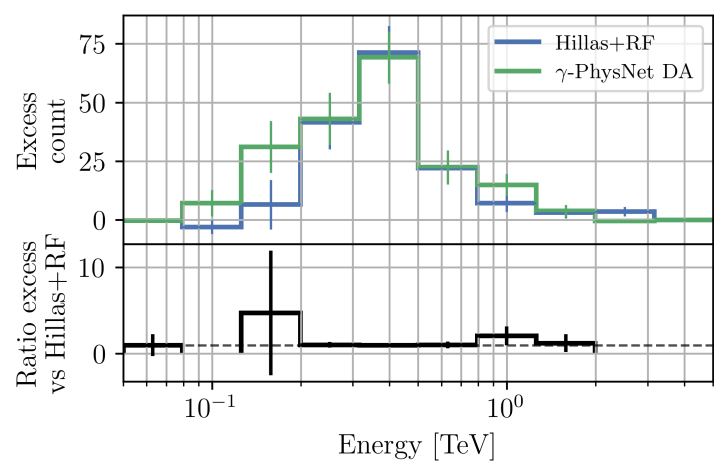

Fig. 5: Excess and relative excess between $\gamma$ PhysNet DA and Hillas+RF as a function of the events reconstructed energy. Bins are missing in the ratio in case of no positive excess for Hillas+RF.

Table I provides the integrated counts and significance for both analysis chains. We can notice that the background levels are similar, as imposed by the procedure. We show here that both analysis chains are able to detect Mrk501, with a slight advantage for $\gamma$-PhysNet DA, showing an integrated excess greater by $30 \%( \pm 23 \%)$ and a higher significance than the reference.

\begin{tabular}{|c|c|c|c|}
\hline Reconstruction & Excess & Significance & Background counts \\
\hline Hillas+RF & $148.7 \gamma$ & $7.6 \sigma$ & 238.3 \\
$\gamma$-PhysNet DA & $192.7 \gamma$ & $9.8 \sigma$ & 226.3 \\
\hline
\end{tabular}

TABLE I: Excess and significance results for Markarian 501.

\section{IV-C Detection of the Crab Nebula}

Here we analyze two observation runs, \#2012 (OFF run) and \#2013 (ON run) taken in February 2020 at zenith angles of $21.4^{\circ}$ and $28.9^{\circ}$ respectively. Both runs undergo the same data reduction as described in section III.

The night sky background (NSB) in the Crab Nebula sky region is much higher than in the case of Mrk 501 and more importantly, much higher than the standard NSB used in simulations. As this can negatively impact the analysis, especially in the case of CNNs which are very sensitive to the noise distribution in the images, we add a poissonian noise to the simulated data. Thanks to Raikov's theorem [25], we determine the parameter of the Poisson distribution as the difference between the simulated average noise pixel charge and the observed one. We can see that

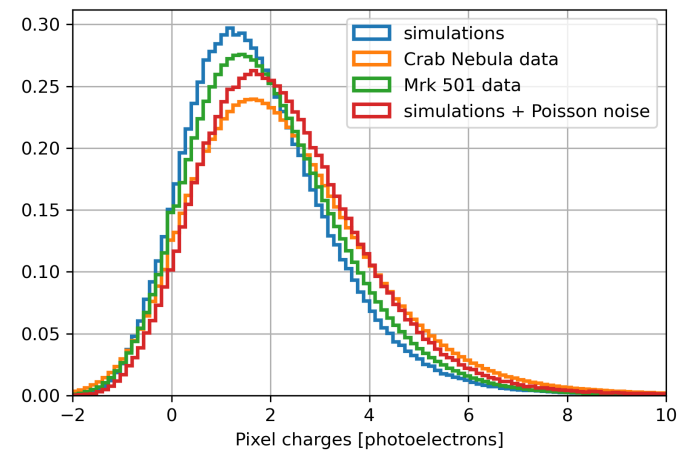

Fig. 6: Distribution of the charges (in photoelectrons) from NSB in the simulations, compared to the one from LST-1 observation data. 


\begin{tabular}{|c|c|c|c|}
\hline & Excess & Significance & Background \\
\hline Hillas+RF & 379 & $12.0 \sigma$ & 308 \\
\hline $\begin{array}{c}\text { Hillas+RF } \\
+ \text { Poisson noise }\end{array}$ & 376 & $11.9 \sigma$ & 305 \\
\hline$\gamma$-PhysNet DA & 395 & $12.5 \sigma$ & 302 \\
\hline $\begin{array}{c}\gamma \text {-PhysNet DA } \\
+ \text { Poisson noise }\end{array}$ & $\mathbf{4 7 6}$ & $\mathbf{1 4 . 3} \sigma$ & 317 \\
\hline
\end{tabular}

TABLE II: Excess and significance results for Crab Nebula.

the additional noise leads to a better agreement between simulated and observed NSB distributions in figure 6. All models are then retrained with these modified simulations.

Here the standard Hillas $+R F$ (without added noise) serves as a reference for the rest of the analysis and is used for background matching to all others analysis as described in section IV-A.

Figure 7 presents the normalized distributions of the angular separation and figure 8 the excess for all analysis chains. One can see here again the higher excess detected by $\gamma$-PhysNet DA compared to Hillas+RF, especially at low energies.

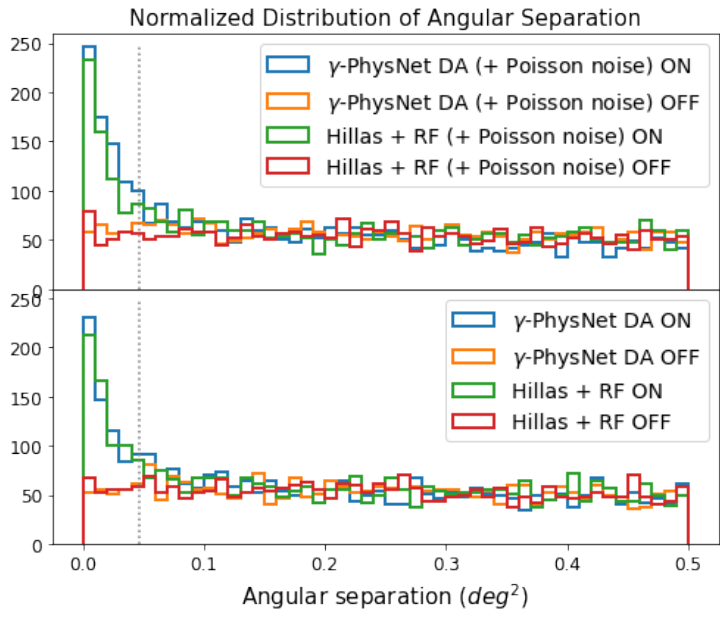

Fig. 7: Gamma-ray event distribution in both observations as a function of the squared angular separation between the reconstructed and the true directions.

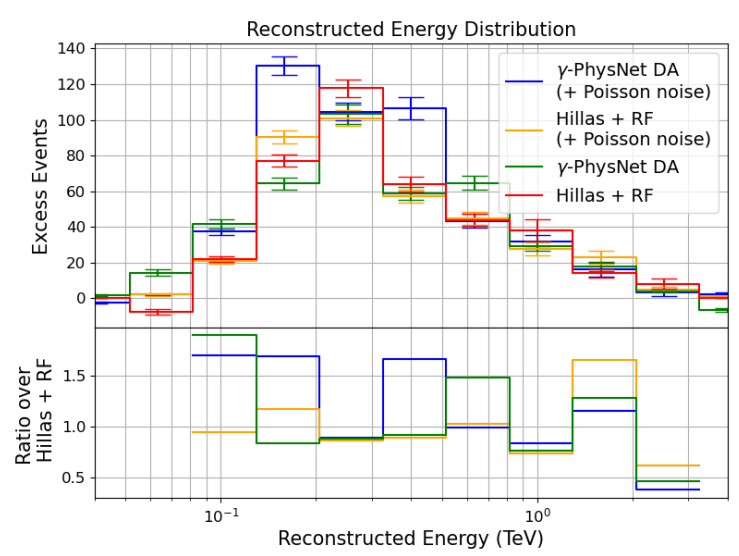

Fig. 8: Excess of detected gamma rays per energy bin. The lower part of the plot represents the ratio of gamma-ray excess per energy bin detected by the models over the one detected by the baseline Hillas+RF method.

Table II displays the final results on Crab Nebula with the excess count, background count and significance. There are several things worth noticing here. First, one can see the higher significance of $\gamma$-PhysNet DA in its first version $(12.5 \sigma)$ compared to Hillas+RF $(12.0 \sigma)$. Then one can see the important improvement brought by the addition of poissonian noise to the simulated dataset, with a final significance of $14.3 \sigma$. Finally, one can note the higher robustness of the Hillas+RF analysis, obtaining very similar results (significance of $11.9 \sigma$ ) after the addition of noise. This is most probably due to the tailcut cleaning applied in this chain which is removing the NSB, thus trading loss of information with robustness. 


\section{Conclusion}

After showing better performances on simulated data, the present study confirms the better sensitivity, especially at low energies, of $\gamma$-PhysNet DA compared to the classical Hillas+RF reconstruction on observations, with two sources detected with higher significance. The greater gain at low energy can easily be explained by the fact that images are on average noisier at these energies and that convolutional neural networks are more powerful at extracting signal in this case. We also show the importance of closely matching the simulated data with the observed ones taking the NSB as use-case, especially in the case of CNNs which are more sensitive to differences between training and test data. The difference in pointing direction between training data and observation is also expected to play a role and its impact on performances will be studied in a later work. This work shows that CNNs are a valid approach for LST-1 event reconstruction, even though it is still in commissioning phase. As shown, their performances can still be improved by a closer match between simulations and data, which will be further refined by our increased understanding of the telescope.

We gratefully acknowledge support from the agencies and organizations listed here: www.ctaobservatory.org/consortium_acknowledgment and here: https://gammalearn.pages.in2p3.fr/page s/acknowledgements/.

\section{References}

[1] L. Breiman, "Random Forests." Machine Learning, vol. 45, pp. 5-32, Jan. 2001.

[2] D. Heck et al., "Corsika: A monte carlo code to simulate extensive air showers," Report fzka, vol. $6019,1998$.

[3] K. Bernlöhr, "Simulation of imaging atmospheric Cherenkov telescopes with CORSIKA and sim_telarray," Astroparticle Physics, vol. 30, pp. 149-158, 2008.

[4] A. Krizhevsky et al., "Imagenet classification with deep convolutional neural networks," Commun. ACM, vol. 60 , 2017.

[5] J. Gu et al., "Recent Advances in Convolutional Neural Networks," arXiv e-prints, p. arXiv:1512.07108, Dec. 2015.

[6] D. Nieto Castaño et al., "Exploring deep learning as an event classification method for the Cherenkov Telescope Array," in 35th ICRC, ser. ICRC, vol. 301, Jan. 2017, p. 809.

[7] D. Nieto Castaño et al., "Reconstruction of iact events using deep learning techniques with ctlearn," in $A D A S S X X X, 2020$.

[8] M. Jacquemont et al., "Single imaging atmospheric cherenkov telescope full-event reconstruction with a deep multi-task learning architecture," in ADASS XXX, 2020.

[9] I. Shilon et al., "Application of deep learning methods to analysis of imaging atmospheric cherenkov telescopes data," Astroparticle Physics, vol. 105, pp. 44-53, 2019.

[10] R. D. Parsons and S. Ohm, "Background rejection in atmospheric Cherenkov telescopes using recurrent convolutional neural networks," European Physical Journal C, vol. 80, p. 363, May 2020.

[11] Kobayashi, Y. et al., "Camera calibration of the cta-lst prototype," in these proceedings.

[12] R. Lopez-Coto et al. for CTA LST project, "lstchain: An analysis pipeline for LST-1, the first prototype Large-Sized Telescope of CTA," in ADASS XXX, 2021.

[13] K. Kosack et al., "cta-observatory/ctapipe: v0.10.5,” Mar. 2021.

[14] M. Nöthe et al., "Prototype open event reconstruction pipeline for the cherenkov telescope array," in these proceedings.

[15] A. Hillas, "Cerenkov light images of eas produced by primary gamma," in ICRC, vol. 3, 1985.

[16] M. Jacquemont et al., "Multi-task architecture with attention for imaging atmospheric cherenkov telescope data analysis," in Proceedings of the 16th VISIGRAPP, INSTICC. SciTePress, 2021.

[17] M. Jacquemont et al., "First Full-Event Reconstruction from Imaging Atmospheric Cherenkov Telescope Real Data with Deep Learning," arXiv e-prints, p. arXiv:2105.14927, May 2021.

[18] K. He et al., "Deep residual learning for image recognition," in 2016 IEEE Conference on Computer Vision and Pattern Recognition (CVPR), 2016, pp. 770-778.

[19] M. Jacquemont et al., "Indexed operations for non-rectangular lattices applied to convolutional neural networks," in Proceedings of the 14th VISIGRAPP - Volume 5: VISAPP, INSTICC. SciTePress, 2019, pp. 362-371.

[20] J. Sun et al., "Saunet: Shape attentive u-net for interpretable medical image segmentation," arXiv preprint arXiv:2001.07645.

[21] Gueta, O. et al., "The cherenkov telescope array: layout, design and performances," in these proceedings, no. $962,2021$.

[22] M. Nöthe et al. (2020, Nov.) pyirf.

[23] Berge, D. et al., "Background modelling in very-high-energy gamma-ray astronomy," A\&A, vol. 466, pp. 1219-1229, 2007.

[24] C. Nigro et al., "Towards open and reproducible multi-instrument analysis in gamma-ray astronomy," , vol. 625, May 2019.

[25] D. Raikov, "On the decomposition of poisson laws," in Dokl. Akad. Nauk SSSR, vol. 14, no. 1, 1937 , pp. 9-12. 


\section{Full Authors List: CTA LST project}

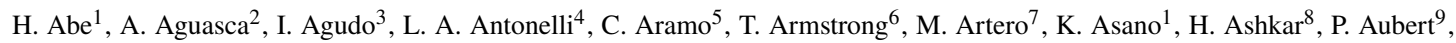
A. Baktash ${ }^{10}$, A. Bamba ${ }^{11}$, A. Baquero Larriva ${ }^{12}$, L. Baroncelli ${ }^{13}$, U. Barres de Almeida ${ }^{14}$, J. A. Barrio ${ }^{12}$, I. Batkovic ${ }^{15}$, J. Becerra González $^{16}$, M. I. Bernardos ${ }^{15}$, A. Berti ${ }^{17}$, N. Biederbeck ${ }^{18}$, C. Bigongiari ${ }^{4}$, O. Blanch ${ }^{7}$, G. Bonnoli ${ }^{3}$, P. Bordas ${ }^{2}$, D. Bose ${ }^{19}$, A. Bulgarelli $^{13}$, I. Burellii ${ }^{20}$, M. Buscemi ${ }^{21}$, M. Cardillo22, S. Caroff ${ }^{9}$, A. Carosi ${ }^{23}$, F. Cassol ${ }^{6}$, M. Cerruti ${ }^{2}$, Y. Chai ${ }^{17}$, K. Cheng ${ }^{1}$, M. Chikawa $^{1}$, L. Chytka ${ }^{24}$, J. L. Contreras ${ }^{12}$, J. Cortina ${ }^{25}$, H. Costantini ${ }^{6}$, M. Dalchenko ${ }^{23}$, A. De Angelis ${ }^{15}$, M. de Bony de Lavergne ${ }^{9}$, G. Deleglise ${ }^{9}$, C. Delgado ${ }^{25}$, J. Delgado Mengual ${ }^{26}$, D. della Volpe ${ }^{23}$, D. Depaoli ${ }^{27,28}$, F. Di Pierro ${ }^{27}$, L. Di Venere ${ }^{29}$, C. Díaz ${ }^{25}$, R. M. Dominik ${ }^{18}$, D. Dominis Prester ${ }^{30}$, A. Donini ${ }^{7}$, D. Dorner ${ }^{31}$, M. Doro ${ }^{15}$, D. Elsässer ${ }^{18}$, G. Emery ${ }^{23}$, J. Escudero ${ }^{3}$, A. Fiasson ${ }^{9}$, L. Foffano ${ }^{23}$, M. V. Fonseca ${ }^{12}$, L. Freixas Coromina ${ }^{25}$, S. Fukami ${ }^{1}$, Y. Fukazawa ${ }^{32}$, E. Garcia ${ }^{9}$, R. Garcia López ${ }^{16}$, N. Giglietto ${ }^{33}$ F. Giordano ${ }^{29}$, P. Gliwny ${ }^{34}$, N. Godinovic ${ }^{35}$, D. Green ${ }^{17}$, P. Grespan ${ }^{15}$, S. Gunji ${ }^{36}$, J. Hackfeld ${ }^{37}$, D. Hadasch ${ }^{1}$, A. Hahn ${ }^{17}$, T.

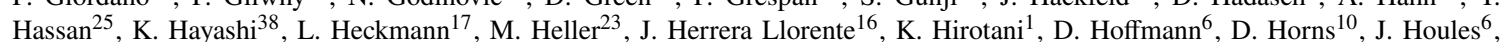
M. Hrabovsky ${ }^{24}$, D. Hrupec ${ }^{39}$, D. Hui ${ }^{1}$, M. Hütten ${ }^{17}$, T. Inada ${ }^{1}$, Y. Inome ${ }^{1}$, M. Iori ${ }^{40}$, K. Ishio ${ }^{34}$, Y. Iwamura ${ }^{1}$, M. Jacquemont ${ }^{9}$, I. Jimenez Martinez $^{25}$, L. Jouvin ${ }^{7}$, J. Jurysek ${ }^{41}$, M. Kagaya ${ }^{1}$, V. Karas ${ }^{42}$, H. Katagiri ${ }^{43}$, J. Kataoka ${ }^{44}$, D. Kerszberg ${ }^{7}$, Y. Kobayashi ${ }^{1}$ A. Kong ${ }^{1}$, H. Kubo ${ }^{45}$, J. Kushida ${ }^{46}$, G. Lamanna ${ }^{9}$, A. Lamastra ${ }^{4}$, T. Le Flour ${ }^{9}$, F. Longo ${ }^{47}$, R. López-Coto ${ }^{15}$, M. López-Moya ${ }^{12}$, A. López-Oramas ${ }^{16}$, P. L. Luque-Escamilla ${ }^{48}$, P. Majumdar ${ }^{19,1}$, M. Makariev ${ }^{49}$, D. Mandat ${ }^{50}$, M. Manganaro ${ }^{30}$, K. Mannheim ${ }^{31}$,

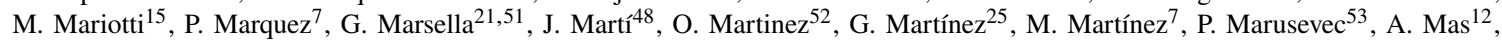
G. Maurin ${ }^{9}$, D. Mazin ${ }^{1,17}$, E. Mestre Guillen ${ }^{54}$, S. Micanovic ${ }^{30}$, D. Miceli ${ }^{9}$, T. Miener ${ }^{12}$, J. M. Miranda ${ }^{52}$, L. D. M. Miranda ${ }^{23}$, R. $^{2}$ Mirzoyan $^{17}$, T. Mizuno ${ }^{55}$, E. Molina' 2 , T. Montaruli ${ }^{23}$, I. Monteiro ${ }^{9}$, A. Moralejo ${ }^{7}$, D. Morcuende ${ }^{12}$, E. Moretti ${ }^{7}$, A. Morselli ${ }^{56}$, K. $^{5}$.

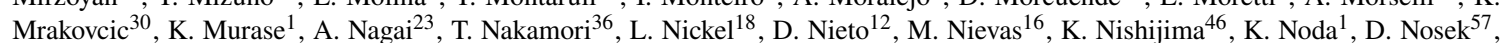
M. Nöthe ${ }^{18}$, S. Nozaki ${ }^{45}$, M. Ohishi ${ }^{1}$, Y. Ohtani ${ }^{1}$, T. Oka ${ }^{45}$, N. Okazaki ${ }^{1}$, A. Okumura ${ }^{58,59}$, R. Orito ${ }^{60}$, J. Otero-Santos ${ }^{16}$, M. Palatiello $^{20}$, D. Paneque ${ }^{17}$, R. Paoletti ${ }^{61}$, J. M. Paredes ${ }^{2}$, L. Pavletić ${ }^{30}$, M. Pech $^{50,62}$, M. Pecimotika $^{30}$, V. Poireau ${ }^{9}$, M. Polo $^{25}$, E. Prandini $^{15}$, J. Prast ${ }^{9}$, C. Priyadarshi ${ }^{7}$, M. Prouza ${ }^{50}$, R. Rando ${ }^{15}$, W. Rhode ${ }^{18}$, M. Ribó ${ }^{2}$, V. Rizi ${ }^{63}$, A. Rugliancich ${ }^{64}$, J. E. Ruiz ${ }^{3}$, T. Saito $^{1}$, S. Sakurai ${ }^{1}$, D. A. Sanchez ${ }^{9}$, T. Šarić ${ }^{35}$, F. G. Saturni ${ }^{4}$, J. Scherpenberg ${ }^{17}$, B. Schleicher ${ }^{31}$, J. L. Schubert ${ }^{18}$, F. Schussler ${ }^{8}$, T. Schweizer $^{17}$, M. Seglar Arroyo ${ }^{9}$, R. C. Shellard ${ }^{14}$, J. Sitarek ${ }^{34}$, V. Sliusar ${ }^{41}$, A. Spolon ${ }^{15}$, J. Striškovićc ${ }^{39}$, M. Strzys ${ }^{1}$, Y. Suda ${ }^{32}$, Y. Sunada $^{65}$, H. Tajima ${ }^{58}$, M. Takahashi ${ }^{1}$, H. Takahashi ${ }^{32}$, J. Takata ${ }^{1}$, R. Takeishi ${ }^{1}$, P. H. T. Tam ${ }^{1}$, S. J. Tanaka ${ }^{66}$, D. Tateishi ${ }^{65}$, L. A.

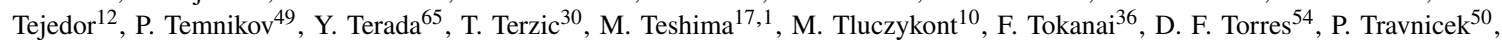
S. Truzzi ${ }^{61}$, M. Vacula ${ }^{24}$, M. Vázquez Acosta ${ }^{16}$, V. Verguilov ${ }^{49}$, G. Verna ${ }^{6}$, I. Viale ${ }^{15}$, C. F. Vigorito ${ }^{27,28}$, V. Vitale ${ }^{56}$, I. Vovk $^{1}, \mathrm{~T}$

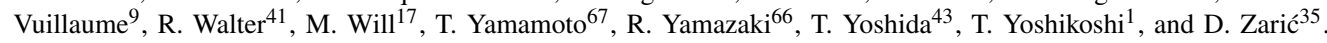

${ }^{1}$ Institute for Cosmic Ray Research, University of Tokyo. ${ }^{2}$ Departament de Física Quàntica i Astrofísica, Institut de Ciències del Cosmos, Universitat de Barcelona, IEEC-UB. ${ }^{3}$ Instituto de Astrofísica de Andalucía-CSIC. ${ }^{4}$ INAF - Osservatorio Astronomico di Roma. ${ }^{5}$ INFN Sezione di Napoli. ${ }^{6}$ Aix Marseille Univ, CNRS/IN2P3, CPPM. ${ }^{7}$ Institut de Fisica d'Altes Energies (IFAE), The Barcelona Institute of Science and Technology. ${ }^{8}$ IRFU, CEA, Université Paris-Saclay. ${ }^{9}$ LAPP, Univ. Grenoble Alpes, Univ. Savoie Mont Blanc, CNRS-IN2P3, Annecy. ${ }^{10}$ Universität Hamburg, Institut für Experimentalphysik. ${ }^{11}$ Graduate School of Science, University of Tokyo. ${ }^{12}$ EMFTEL department and IPARCOS, Universidad Complutense de Madrid. ${ }^{13}$ INAF - Osservatorio di Astrofisica e Scienza dello spazio di Bologna. ${ }^{14}$ Centro Brasileiro de Pesquisas Físicas. ${ }^{15}$ INFN Sezione di Padova and Università degli Studi di Padova. ${ }^{16}$ Instituto de Astrofísica de Canarias and Departamento de Astrofísica, Universidad de La Laguna. ${ }^{17}$ MaxPlanck-Institut für Physik. ${ }^{18}$ Department of Physics, TU Dortmund University. ${ }^{19}$ Saha Institute of Nuclear Physics. ${ }^{20}$ INFN Sezione di Trieste and Università degli Studi di Udine. ${ }^{21}$ INFN Sezione di Catania. ${ }^{22}$ INAF - Istituto di Astrofisica e Planetologia Spaziali (IAPS). ${ }^{23}$ University of Geneva - Département de physique nucléaire et corpusculaire. ${ }^{24}$ Palacky University Olomouc, Faculty of Science. ${ }^{25}$ CIEMAT. ${ }^{26}$ Port d'Informació Científica. ${ }^{27}$ INFN Sezione di Torino. ${ }^{28}$ Dipartimento di Fisica - Universitá degli Studi di Torino. ${ }^{29}$ INFN Sezione di Bari and Università di Bari. ${ }^{30}$ University of Rijeka, Department of Physics. ${ }^{31}$ Institute for Theoretical Physics and Astrophysics, Universität Würzburg. ${ }^{32}$ Physics Program, Graduate School of Advanced Science and Engineering, Hiroshima University. ${ }^{33}$ INFN Sezione di Bari and Politecnico di Bari. ${ }^{34}$ Faculty of Physics and Applied Informatics, University of Lodz. ${ }^{35}$ University of Split, FESB. ${ }^{36}$ Department of Physics, Yamagata University. ${ }^{37}$ Institut für Theoretische Physik, Lehrstuhl IV: Plasma-Astroteilchenphysik, Ruhr-Universität Bochum. ${ }^{38}$ Tohoku University, Astronomical Institute. ${ }^{39}$ Josip Juraj Strossmayer University of Osijek, Department of Physics. ${ }^{40}$ INFN Sezione di Roma La Sapienza. ${ }^{41}$ Department of Astronomy, University of Geneva. ${ }^{42}$ Astronomical Institute of the Czech Academy of Sciences. ${ }^{43}$ Faculty of Science, Ibaraki University. ${ }^{44}$ Faculty of Science and Engineering, Waseda University. ${ }^{45}$ Division of Physics and Astronomy, Graduate School of Science, Kyoto University. ${ }^{46}$ Department of Physics, Tokai University. ${ }^{47}$ INFN Sezione di Trieste and Università degli Studi di Trieste. ${ }^{48}$ Escuela Politécnica Superior de Jaén, Universidad de Jaén. ${ }^{49}$ Institute for Nuclear Research and Nuclear Energy, Bulgarian Academy of Sciences. ${ }^{50} \mathrm{FZU}$ - Institute of Physics of the Czech Academy of Sciences. ${ }^{51}$ Dipartimento di Fisica e Chimica 'E. Segrè' Università degli Studi di Palermo. ${ }^{52}$ Grupo de Electronica, Universidad Complutense de Madrid. ${ }^{53}$ Department of Applied Physics, University of Zagreb. ${ }^{54}$ Institute of Space Sciences (ICE-CSIC), and Institut d'Estudis Espacials de Catalunya (IEEC), and Institució Catalana de Recerca I Estudis Avançats (ICREA). ${ }^{55}$ Hiroshima Astrophysical Science Center, Hiroshima University. ${ }^{56}$ INFN Sezione di Roma Tor Vergata. ${ }^{57}$ Charles University, Institute of Particle and Nuclear Physics. ${ }^{58}$ Institute for Space-Earth Environmental Research, Nagoya University. ${ }^{59}$ Kobayashi-Maskawa Institute (KMI) for the Origin of Particles and the Universe, Nagoya University. ${ }^{60}$ Graduate School of Technology, Industrial and Social Sciences, Tokushima University. ${ }^{61}$ INFN and Università degli Studi di Siena, Dipartimento di Scienze Fisiche, della Terra e dell'Ambiente (DSFTA). ${ }^{62}$ Palacky University Olomouc, Faculty of Science. ${ }^{63}$ INFN Dipartimento di Scienze Fisiche e Chimiche - Università degli Studi dell'Aquila and Gran Sasso Science Institute. ${ }^{64}$ INFN Sezione di Pisa. ${ }^{65}$ Graduate School of Science and Engineering, Saitama University. ${ }^{66}$ Department of Physical Sciences, Aoyama Gakuin University. ${ }^{67}$ Department of Physics, Konan University. 